\title{
TETRACYCLINE RESISTANT AND POLYMYXIN B SENSITIVE VIBRIO CHOLERAE O1 EL TOR ISOLATED FROM THE RECENT EPIDEMICS
}

\author{
MasaAki IWANAGA ${ }^{1}$, Sithat InsisiengmaY ${ }^{2}$, NAOMI HigA ${ }^{1}$ \\ AND LAY SISAVATH ${ }^{2}$ \\ Received August 16, 1999/Accepted October 4, 1999
}

\begin{abstract}
By examining 99 Vibrio cholerae $\mathrm{O} 1$ isolates from cholera epidemics in a variety of areas in Laos in 1998, we found two unusual characteristics of the organisms. All except 4 isolates were moderately resistant to tetracycline without having plasmids, and susceptibilities to the other drugs were as expected. Eleven isolates showed the same level of susceptibility to polymyxin B as classical $V$. cholerae O1. With the exception of 4 tetracycline-susceptible strains, all the isolates were resistant to or poorly sensitive to the vibriostatic agent $\mathrm{O} / 129$ (2,4-diamino-6,7-di-iso-propyl pteridine phosphate). All isolates, including those susceptible to polymyxin B, produced E1 Tor hemolysin which was neutralized by anti-E1 Tor hemolysin and were resistant to cholera phage IV, indicating that they were of the E1 Tor biotype.
\end{abstract}

Key words: Vibrio cholerae O1, tetracycline, polymyxin B, drug sensitivity, Laos

\section{INTRODUCTION}

The current cholera pandemic, which began in 1961 from a focus in South Sulawesi, Indonesia, shows no signs of declining. During the past 4 decades, there have been a variety of changes in the epidemic cholera vibrios; these changes include hemolytic properties of $\mathrm{E} 1$ Tor strains, drug susceptibilities, disappearance and reappearance of classical cholera vibrios and the appearance of new serovar $V$. cholerae O139. Tetracycline was originally the first choice for antibiotic therapy and prophylaxis of cholera. The first epidemic due to a tetracycline resistant strain was seen in Tanzania in 1977 (Mhalu et al., 1979). Soon after this, a tetracycline resistant strain caused an outbreak in Bangladesh (Glass et al., 1980). The vibrios isolated in both countries were plasmid-mediated multiple drug-resistant strains. The first outbreak in Tanzania was long-lasting and spread to Kenya around Lake Victoria (Ehara et al., 1983), whereas the outbreak in Bangladesh was limited to the Matrab area and disappeared after 5 months. The emergence of drug-resistant strains in Tanzania was attributed to extensive use of tetracycline for prophylaxis (Towner et al., 1980). In Bangladesh, however, prophylaxis was not common, and the source of outbreak could not be determined. Although a few strains of drug-resistant $V$. cholerae $\mathrm{O} 1$ have been reported (Kobari et al., 1970; Threlfall et al., 1993; Yamamoto et al., 1995), there have been no large outbreaks other than these two episodes. In 1998, cholera outbreaks due to tetracycline-resistant strains occurred in a wide area of Laos. Here, we describe characterization of the $V$. cholerae $\mathrm{O} 1$ isolated in Laos in 1998.

\section{Materials AND Methods}

Bacterial strains: A total of $99 \mathrm{~V}$. cholerae $\mathrm{O} 1$ strains isolated from patients in Laos in 1998 were

1 Department of Bacteriology, Faculty of Medicine, University of the Ryukyus, 207 Uehara, Nishihara, Okinawa 903-0215, Japan

2 Center for Laboratory and Epidemiology, Km3, Thadeua Rd., Vientiane, Lao People's Democratic Republic Corresponding author: MASAAKI IWANAGA

Mailing address: Department of Bacteriology, Faculty of Medicine, University of the Ryukyus, 207 Uehara, Nishihara, Okinawa 903-0215, Japan

Phone: +81-98-895-3331 Ext. 2285

FAX: $+81-98-895-2951$

E-mail: 
Table 1 Characteristics of isolates and the places of isolation

\begin{tabular}{lcccc}
\hline Place & $\begin{array}{c}\text { number of } \\
\text { isolates }\end{array}$ & $\begin{array}{c}\text { resistant to } \\
\text { tetracycline }\end{array}$ & $\begin{array}{c}\text { resistant to } \\
\text { O/129* }\end{array}$ & $\begin{array}{c}\text { sensitive to } \\
\text { polymyxin B }\end{array}$ \\
\hline Vientiane & 82 & 78 & 77 & 3 \\
\hline Xaisettha & 43 & 39 & 39 & 2 \\
Xaithani & 30 & 30 & 29 & 1 \\
Sisathanak & 5 & 5 & 5 & 0 \\
Thaluang & 2 & 2 & 2 & 0 \\
Thourakham & 1 & 1 & 1 & 0 \\
Maxaythang & 1 & 1 & 1 & 0 \\
\hline Khammouan & 9 & 9 & 9 & 0 \\
Savanakhet & 4 & 4 & 4 & 4 \\
Champasak & 4 & 4 & 4 & 4 \\
\hline Total & 99 & 95 & 94 & 11 \\
\hline
\end{tabular}

*includes moderately resistant strains

examined. The strains were isolated during the period from May to November 1998 from a wide area in the Southern half of the country as shown in Table 1.

Identification of $V$. cholerae $\mathrm{O} 1$ : The isolates were identified as $V$. cholerae $\mathrm{O} 1$ by routine laboratory tests. Serotype was determined by the slide agglutination method using commercial anti-Ogawa and anti-Inaba sera (Denkaseiken Co. Tokyo).

Production of and susceptibility to phage: Suscepti bility to cholera phage IV, E1 Tor phage 5, kappaphage, fs- 1 and fs- 2 phage was examined by the following method. First, $10 \mathrm{~m} l$ of peptone agar (1\% peptone, $0.5 \% \mathrm{NaCl}, 1.5 \%$ agar) was poured into a Petri dish (diameter, $9 \mathrm{~cm}$ ) and solidified. Overnight heart infusion broth culture of each isolate $(0.2 \mathrm{ml})$ and $4 \mathrm{ml}$ of soft agar ( $1 \%$ peptone, $0.5 \% \mathrm{NaCl}, 0.5 \%$ yeast extract, $0.6 \%$ agar) kept at $43^{\circ} \mathrm{C}$ were mixed and poured on to peptone agar plates. Aliquot of $10 \mu l$ of phage solution at the routine test dilution were spotted on to the soft agar plates containing the organisms. After $24 \mathrm{hr}$ incubation at $37^{\circ} \mathrm{C}$, the vibrios showing plaque formation were regarded as susceptible strains. Production of kappa phage by the organisms was examined as described by Takeya and Shimodori (1963). Briefly, broth cultures of each isolate were treated with chloroform to kill the bacterial cells. The killed culture fluid $(0.1 \mathrm{~m} l)$, a $0.2 \mathrm{~m} l$ broth culture of an indicator strain H218 ( $V$. cholerae O1, serotype Ogawa, biotype cholerae) and 4 $\mathrm{m} l$ soft agar kept at $43^{\circ} \mathrm{C}$ were mixed and poured on to peptone agar plates. After overnight incubation at $37^{\circ} \mathrm{C}$, plaque formation was observed. To examine productivity of filamentous phage fs -1 and fs -2 , the organisms were cultured in HIB with shaking overnight and the cell-free supernatant was spotted on to the indicator strain UDT109 ( $V$. cholerae O1 E1 Tor, Inaba).
Cholera toxin production: The organisms were cultured in AKI medium at $37^{\circ} \mathrm{C}$ for $20 \mathrm{hr}$ in a stationary test tube, after which the cholera toxin in the culture supernatant was titrated using the reversed passive latex agglutination method (Iwanaga and Yamamoto, 1985).

Hemolysin production: The isolates were inoculated on to sheep blood agar plates, and hemolysis around the grown colonies was examined. The isolates were also cultured in heart infusion broth supplemented with $1 \%$ glycerol and incubated at $37^{\circ} \mathrm{C}$ for $24 \mathrm{hr}$. Washed sheep red blood cells at the final concentration of $0.5 \%$ were added to the culture supernatant, incubated at $37^{\circ} \mathrm{C}$ for $2 \mathrm{hr}$ followed by overnight incubation in a cold room. Neutralization test was carried out by adding 1 to 20 volumes of anti-E1 Tor hemolysin serum to the culture supernatant.

Drug susceptibilities: Susceptibilities of the isolates to ampicillin (ABPC, Meiji), tetracycline (TC, Wako), erythromycin (EM, Dainihon), ofloxacin (OFLX, Daiichi) and polymyxin $\mathrm{B}(\mathrm{PmxB}$, Feizer) were determined by multipoint inoculation onto a series of heart infusion agar plates containing doubling dilutions of the drugs from $100 \mu \mathrm{g} / \mathrm{m} l$ to $0.0125 \mu \mathrm{g} / \mathrm{m} l$. A 10 -fold dilution of overnight broth culture was inoculated in to each plate using Microplanter (Sakuma Co. model MITP \# 00257). The susceptibility was expressed as minimum inhibitory concentration (MIC) of each drug.

Isolation of plasmid and electrophoresis: Plasmid DNA was isolated by the method of Kado and Liu (1981) with slight modifications (lysing buffer was added and stand still at room temperatue before phenol extraction) and electrophoresed in $0.8 \%$ agarose gel. The gels were stained with ethidium bromide and observed under a UV transilluminator.

\section{RESULTS}

All isolates revealed typical biochemical characteristics of $V$. cholerae, but 9 out of the 99 strains did not grow in peptone water without sodium chloride, and 19 strains were negative for Voges-Proskauer reaction. All isolates were toxigenic, and hemolytic on blood agar plates, and the hemolysis was inhibited by anti-E1 Tor hemolysin serum. All isolates belonged to serotype Ogawa.

Phage: Ninety-six of the 99 isolates were susceptible to E1 Tor phage 5, and none was susceptible to cholera phage IV. Kappa phage was produced by 96 isolates. The remaining 3 non-producers were susceptible to this phage; i.e. all belong to the Celebes type 
Table 2 Production of and susceptibility to phages

\begin{tabular}{lcccc}
\hline phage & productive & sensitive & independent & Total \\
\hline cholera IV & 0 & 0 & 99 & 99 \\
E1 Tor 5 & 0 & 96 & 3 & 99 \\
kappa & 96 & 3 & 0 & 99 \\
fs-1 & 0 & 3 & 96 & 99 \\
fs-2 & 0 & 7 & 92 & 99 \\
\hline
\end{tabular}

Table 3 Drug susceptibilities of 99 isolates

\begin{tabular}{cccccc}
\hline$\mu \mathrm{g} / \mathrm{m} l$ & TC & ABPC & EM & OFLX & Pmx-B \\
\hline $0.0125 \geqq$ & 0 & 0 & 0 & 86 & 0 \\
0.025 & 0 & 0 & 0 & 13 & 0 \\
0.05 & 0 & 0 & 0 & 0 & 0 \\
0.1 & 0 & 0 & 0 & 0 & 0 \\
0.2 & 1 & 0 & 0 & 0 & 0 \\
0.39 & 3 & 0 & 0 & 0 & 2 \\
0.78 & 0 & 0 & 0 & 0 & 8 \\
1.56 & 0 & 1 & 0 & 0 & 1 \\
3.13 & 54 & 26 & 38 & 0 & 0 \\
6.25 & 41 & 72 & 61 & 0 & 0 \\
12.5 & 0 & 0 & 0 & 0 & 0 \\
25 & 0 & 0 & 0 & 0 & 0 \\
50 & 0 & 0 & 0 & 0 & 7 \\
100 & 0 & 0 & 0 & 0 & 80 \\
$100<$ & 0 & 0 & 0 & 0 & 1 \\
\hline
\end{tabular}

TC: tetracycline, ABPC: ampicillin, EM: erythromycine, OFLX: ofloxacine, Pmx-B: polymyxin B

including 3 cured Celebes. Three and 7 of the 99 isolates were susceptible to the filamentous phage fs- 1 and $\mathrm{fs}-2$, respectively. None of the strains produced fs -1 or fs -2 (Table 2).

Drug susceptibilities: Almost all isolates were mod erately resistant to tetracycline, with MIC of 3.13 or $6.25 \mu \mathrm{g} / \mathrm{m} l$. Only 4 isolates from an area of Vientiane were sensitive to tetracycline with MIC of 0.2 or 0.39 $\mu \mathrm{g} / \mathrm{m} l$. Most of the isolates showed polymyxin B MICs of $100 \mu \mathrm{g} / \mathrm{m} l$, but 11 showed MICs of 0.39 to $1.56 \mu \mathrm{g} / \mathrm{m} l$. Polymyxin B-susceptible strains were isolated from Vientiane (3 of 80 isolates), Savanakhet (4 of 4) and Champasak (4 of 4), areas separated by fairly large distance. The susceptibilities to ampicillin, erythromycin, and ofloxacine were similar to the expected values (Tables 2 and 3 ).

Susceptibility to O/129: The size of the zone of growth inhibition around the disk containing $150 \mathrm{mg}$ of the vibriostatic agent O/129 (2,4-diamino-6,7-di-isopropyl pteridine phosphate) was variable, therefore, the susceptibilities were defined as follows. Resistance was defined as an inhibition zone of 0 to $1 \mathrm{~mm}$, and susceptibility was defined as a zone of more than $10 \mathrm{~mm}$. Other strains were expressed as intermediate. According to these criteria, 31 strains were resistant and 5 (including 4 tetracycline-sensitive strains) were sensitive to $\mathrm{O} / 129$.

Plasmids: No plasmids were detected in 20 randomly selected strains, whereas the tetracycline-resistant strains isolated in Kenya in 1982 examined as positive controls contained plasmids.

\section{DISCUSSION}

The present study revealed 2 novel findings in the drug susceptibility pattern of epidemic $V$. cholerae $\mathrm{O}$. Among the $99 V$. cholerae $\mathrm{O} 1 \mathrm{E} 1 \mathrm{~T}$ or isolated in these epidemics, 11 were sensitive to polymixin B with the same MIC as classical cholera vibrios, and 95 were moderately resistant to tetracycline without harboring any plasmids as far as 20 isolates examined are concerned. The MIC of polymyxin B against $V$. cholerae $\mathrm{O} 1$ E1 Tor is 50 to $200 \mu \mathrm{g} / \mathrm{m} l$, whereas that of classical $V$. cholerae $\mathrm{O} 1$ is 0.4 to $1.56 \mu \mathrm{g} / \mathrm{ml}$. Although we found a strain against which the MIC of polymixin B was 6.25 $\mu \mathrm{g} / \mathrm{m} l$ in the epidemic in 1994 in Laos (Higa et al., 1995), epidemic E1 Tor strains showing the same susceptibilities as classical strains ( 0.4 to $1.56 \mu \mathrm{g} / \mathrm{m} l)$ were found in the present study for the first time. In Southern Laos, all isolates (8 strains) were polymyxin B-sensitive, whereas 3 of 80 isolates from Vientiane, the capital of Laos. $V$. cholerae $\mathrm{O} 1 \mathrm{E} 1 \mathrm{~T}$ Tor were distinguished from classical $V$. cholerae $\mathrm{O} 1$ by their productivity of $\mathrm{E} 1$ Tor hemolysin. However, the susceptibilities of $V$. cholerae $\mathrm{O} 1$ to polymyxin $\mathrm{B}$ and cholera phage IV have been reported to correspond to the productivity of E1 Tor hemolysin (Nakasone et al., 1987). Therefore, these 2 biotypes are usually distinguished by testing susceptibility to polymyxin B. However, the present study suggested that biotype should be determined by analysis of E1 Tor hemolysin productivity and susceptibility ot cholera phage IV.

All strains of tetracycline-resistant $V$. cholerae $\mathrm{O} 1$ reported to date were plasmid-dependent, multi-drug resistant, with a tetracycline MIC of 25 to $200 \mu \mathrm{g} / \mathrm{m} l$ (Coppo et al., 1995; Glass et al., 1980; Towner et al., 1980), except a report by Yamamoto et al. (1995). In contrast, the organisms described here did not harbor any plasmids, and were moderately resistant to tetracycline with MICs of 3.13 or $6.25 \mu \mathrm{g} / \mathrm{m} l$. MICs of EM, $\mathrm{ABPC}$ and OFLX remained within the expected range. These isolates were generally resistant to the vibrio static agent $\mathrm{O} / 129$. Only 5 isolates including 4 tetracycline-sensitive strains were sensitive to $\mathrm{O} / 129$. These observation do not, however, necessarily indicate a close correlation between resistance to $\mathrm{O} / 129$ and tetracycline, as that $V$. cholerae $\mathrm{O} 139$ is resistant to the former but susceptible to the latter (Albert et al., 1997; Higa et al., 1995).

Recently, 83 of 93 isolates (89\%) of tetracycline sensitive $V$. cholerae $\mathrm{O} 1 \mathrm{E} 1 \mathrm{~T}$ Tor collected from a variety 
of areas throughout the world were reported to be lysogenized by the phage fs-2 (Ikema and Honma, 1998). However, in the present study, 92 of 99 isolates of tetracycline resistant $V$. cholerae $\mathrm{O} 1 \mathrm{E} 1 \mathrm{~T}$ or were not associated with fs-2. Considering the phages as the gene vector, the relation between $\mathrm{fS}_{\mathrm{S}}-2$ and drug sensitivities is now under investigation.

The phenotypic changes in these isolates must have been based on chromosomal mutations as seen in the case of sulfamethoxazole-trimethoprim-resistant $V$. cholerae $\mathrm{O} 139$ in which susceptibility was shown to be dependent on chromosomal self-transmissible genetic elements (Waldor et al., 1996), since no plasmid was detected in the examined 20 isolates. The phenotypes based on chromosome would be more stable than that associated with plasmids, therefore, careful monitoring of these mutant organisms is required.

\section{REFERENCES}

1) Albert, M.J., Bhuiyan, N.A., Talukder, K.A., Faruque, A. S.G., Nahar, S., Faruque, S.M., Ansaruzzaman, M. and Rahman, M. (1997): Phenotypic and genotypic changes in Vibrio cholerae O139 Bengal. J. Clin. Microbiol., 35, 2588-2592

2 ) Coppo, A., Colombo, M., Pazzani, C., Bruni, R., Mohamud, K.A., Omar, K.H., Mastrandrea, S., Salvia, A.M., Rotigliano, G. and Maimone, F. (1995): Vibrio cholerae in the horn of Africa: Epidemiology, plasmids, tetracycline resistance gene amplification, and comparison between $\mathrm{O} 1$ and non-O1 strains. Am. J. Trop. Med. Hyg., 53, 351-359

3 ) Ehara, M., Watanabe, S., Ichinose, Y., Shimodori, S., Siongok, T.K.A., Alia, K. and Sang, F.C. (1984): Epidemiological observation of cholera in rural Kenya in 1983. In: Proc. The 20th Joint Conference of cholera; US-Japan Cooperative Medical Science Program. Nov. 608, 1984, Nara, Japan.

4 ) Glass, R.I., Huq, I., Alim, A.R.M.A. and Yunus, M. (1980): Emergence of multiply antibiotic-resistant $\mathrm{Vi}$ brio cholerae in Bangladesh. J. Infect. Dis., 142, 939-942

5 ) Higa, N., Iwanaga, M., Utsunomiya, A, Kuyyakanond,
T., Sithivong, N., Wasito, E.B., Toma, C. and Yamashiro, T. (1995): Drug sensitivity of Vibrio cholerae and Shigella species in the world. Jpn. J. Trop. Med. Hyg. 23, 159-164

6 ) Ikema, M. and Honma, Y. (1998): A novel filamentous phage, fs-2, of Vibrio cholerae O139. Microbiol., 144, 1901-1906

7 ) Iwanaga, M. and Yamamoto, K. (1985): New medium for the production of cholera toxin by Vibrio cholerae $\mathrm{O} 1$ E1 Tor. J. Clin. Microbiol., 22, 405-408

8 ) Kado, C.I. and Liu, S.T. (1981): Rapid procedure for detection and isolation of large and small plasmids. J. Bacteriol., 145, 1365-1375

9 ) Kobari, K., Takakura, I., Nakatomi, M., Sogame, S. and Uylangco, C. (1970): Antibiotic-resistant strains of E1 Tor vibrio in the Philippines and the use of Furalazine for chemotherapy. Bull. Wld. Hlth. Org., 43, 365-371

10) Mhalu, F.S., Mmari, P.W. and Ijumba, J. (1979): Rapid emergence of E1 Tor Vibrio cholerae resistant to antimicrobial agents during the first six months of fourth cholera epidemic in Tanzania. Lancet, 1, 345-347

11) Nakasone N., Iwanaga, M. and Eeckels, R. (1987): Characterization of Vibrio cholerae $\mathrm{O} 1$ recently isolated in Bangladesh. Trans. Roy. Soc. Trop. Med. Hyg., 81, 876-878

12) Takeya, K. and Shimodori, S. (1963): Prophage-typing of E1 Tor vibrios. J. Bacteriol., 85, 957-958

13) Threlfall, E.J., Said, B. and Rowe, B. (1993): Emergence of multiple drug resistance in Vibrio cholerae $\mathrm{O} 1 \mathrm{E} 1 \mathrm{~T}$ Tor from Ecuador. Lancet, 342, 1173

14) Towner, K.J., Pearson, N.J., Mhalu, F.S. and O'Grady, F. (1980): Resistance to antimicrobial agents of Vibrio cholerae E1 Tor strains isolated during the fourth cholera epidemic in the United Republic of Tanzania. Bull. Wld. Hlth. Org., 58, 747-751

15) Waldor, M.K., Tschape, H. and Mekalanos, J.J. (1996): A new type of conjugative transposon encodes resistance to sulfamethoxazole, trimethoprim and streptomycin in Vibrio cholerae O139. J. Bacteriol., 178, 4157-4165

16) Yamamoto, T., Nair, G.B., Albert, M.J., Parodi, C.C. and Takeda, Y. (1995): Survey of in vitro susceptibilities of Vibrio cholerae $\mathrm{O} 1$ and $\mathrm{O} 139$ to antimicrobial agents. Antimicrob. Agents Chemother., 39, 241-244 